\title{
BMJ Open Surgical fixation with K-wires versus plaster casting in the treatment of dorsally displaced distal radius fractures: protocol for Distal Radius Acute Fracture Fixation Trial 2 (DRAFFT 2)
}

Juul Achten, ${ }^{1}$ William Sones, ${ }^{1}$ Joseph Dias, ${ }^{2}$ Helen Hedley, ${ }^{3}$ Jonathan A Cook, ${ }^{1}$ Melina Dritsaki, ${ }^{1}$ May Ee Png, ${ }^{1}$ Alastair Gray, ${ }^{4}$ Sarah E Lamb, ${ }^{1}$ Matthew L Costa ${ }^{1}$

To cite: Achten J, Sones W, Dias J, et al. Surgical fixation with $\mathrm{K}$-wires versus plaster casting in the treatment of dorsally displaced distal radius fractures: protocol for Distal Radius Acute Fracture Fixation Trial 2 (DRAFFT 2). BMJ Open 2019;9:e028474. doi:10.1136/ bmjopen-2018-028474

- Prepublication history for this paper is available online. To view these files, please visit the journal online (http://dx.doi. org/10.1136/bmjopen-2018028474).

Received 11 December 2018 Revised 25 January 2019 Accepted 28 January 2019
D) Check for updates

(c) Author(s) (or their employer(s)) 2019. Re-use permitted under CC BY-NC. No commercial re-use. See rights and permissions. Published by BMJ.

For numbered affiliations see end of article.

\section{Correspondence to}

Professor Matthew L Costa; matthew.costa@ndorms.ox. ac.uk

\begin{abstract}
Introduction Optimal management of distal radius fractures in adults remains controversial. Previous evidence and current clinical guidelines tell us that, if a closed reduction of a dorsally displaced fracture is possible, Kirschner wires (K-wires) are the preferred form of surgical fixation. However, the question remains whether there is any need to perform surgical fixation following a successful closed reduction, or is a simple plaster cast as effective? This is the protocol for a randomised controlled trial of manipulation and surgical fixation with K-wires versus manipulation and casting in the treatment of dorsally displaced distal radius fractures.

Methods and analysis Adult patients with an acute dorsally displaced fracture of the distal radius are potentially eligible to take part. Prior to surgery, baseline demographic data, radiographs, data on pain/ function using the Patient-Rated Wrist Evaluation Score (PRWE) and health-related quality of life (HRQoL) using the EuroQoL 5-dimension 5-level (EQ-5D-5L) will be collected. A randomisation sequence, stratified by centre, intra-articular extension of the fracture and age, will be administered via a secure web-based service. Each patient will be randomly allocated to either 'manipulation and surgical fixation with K-wires' or 'manipulation and plaster casting'. A clinical assessment, radiographs and records of early complications will be recorded at 6 weeks. PRWE and HRQoL outcome data will be collected at 3, 6 and 12 months post-randomisation. Further information will be requested with regard to healthcare resource use and any complications.
\end{abstract}

Ethics and Dissemination The National Research Ethic Committee approved this study on 6 0ctober 2016 (16/ SC/0462). The National Institute for Health Research Health Technology Assessment monograph and a manuscript to a peer-reviewed journal will be submitted on completion of the trial. The results of this trial will substantially inform clinical practice on the clinical and cost-effectiveness of the treatment of this injury. Trial registration number ISRCTN11980540; Pre-results.
Strengths and limitations of this study

Broad eligibility criteria to ensure generalisability.

- Patient-centred outcome data.

- Assessment of outcomes at multiple time points will allow for information on recovery profile.

- In addition to a comparison of clinical outcomes, a health economic evaluation will be performed.

- It will not be possible to blind patients and caregivers to their allocated treatment.

\section{BACKGROUND}

Fractures of the distal radius are extremely common injuries. In the developed world, $6 \%$ of women will have sustained such a fracture by the age of 80 and $9 \%$ by the age of $90 .{ }^{1}$ As the population continues to age, these figures are likely to increase further. The optimal management of fractures of the distal radius in adults remains controversial. There is a bimodal distribution in terms of age. Younger patients frequently sustain complicated, high-energy injuries involving the wrist joint. However, fractures of the distal radius are also common in older patients who are more likely to sustain low-energy fractures related to osteoporosis. ${ }^{2}$ This study is designed to address both groups of patients, as the key management decisions pertain to all patients with a fracture of the distal radius.

In general, if the bone fragments are undisplaced, that is, the bone fragments remain in anatomical alignment, fractures of the distal radius are treated non-operatively. However, if the bone fragments have displaced, that is, moved out of their normal alignment, the treating clinician will usually recommend 
a 'manipulation' of the bone fragments to restore the normal anatomy. Manipulating a fracture is painful, therefore, this is carried out using either local, regional or general anaesthetic.

Following the manipulation, the bone fragments can fall back out of normal alignment. Therefore, the treating clinician will apply support to the bone fragments while they heal.

This trial will compare two techniques for holding the position of the bone fragments following a manipulation of a dorsally displaced fracture of the distal radius; a moulded plaster cast versus surgical fixation with Kirschner wires (K-wires).

Handoll and Madhok ${ }^{3}$ summarised the results of a series of Cochrane reviews of randomised controlled trials of the treatment of fractures of the distal radius and 'exposed the serious deficiency in the available evidence'. Furthermore, they were able to identify key areas for future research including "when and what type of surgery is indicated'. In 2014, we published the results of the Distal Radius Acute Fracture Fixation Trial (DRAFFT; NIHR-HTA 08/116/97). ${ }^{4}$ In this study, we randomly assigned 461 adult patients having surgery for a dorsally displaced fracture of the distal radius to either K-wire fixation or locking-plate fixation. Contrary to the existing literature, and against the increasing use of plate fixation, the DRAFFT study showed that, if a closed reduction of the fracture was possible, there is no difference between K-wires and locking-plates for patients with fractures of the distal radius. K-wire fixation is less expensive and quicker to perform. This evidence led to a change in clinical practice in the $\mathrm{UK},{ }^{5}$ and changes to national guidelines on the management of this injury. ${ }^{6}$

However, there remain unanswered questions; specifically, is there any need to perform surgical fixation of the fracture following a closed reduction of the distal radius, or is a simple plaster cast as effective as the insertion of metalwork?

Therefore, we propose:

A randomised controlled trial of manipulation and surgical fixation with K-wires versus manipulation and casting in the treatment of adult patients with a dorsally displaced fracture of the distal radius.

\section{Good Clinical Practice}

The trial will be carried out in accordance with Medical Research Council Good Clinical Practice and applicable UK legislation using the following protocol (V1.0 9 August 2016).

\section{Consolidated standards of reporting trials}

The trial will be reported in line with the Consolidated Standards of Reporting Trials statement using the non-pharmacological treatment interventions extension.

\section{Aim}

The aim of this project is to improve wrist function and reduce pain by determining the best treatment strategy for adult patients who have sustained a dorsally displaced fracture of the distal radius.

\section{Objectives}

The primary objective is:

To quantify and draw inferences on observed differences in the Patient-Rated Wrist Evaluation (PRWE, a validated assessment of wrist function and pain) between surgical fixation with K-wires versus plaster casting in the first year after the injury.

The secondary objectives are:

1. To quantify and draw inferences on differences in the EuroQoL 5-dimension 5-level (EQ-5D-5L) (a validated assessment of health-related quality of life [HRQoL]) between surgical fixation with K-wires versus plaster casting over the first year post-randomisation.

2. To determine the complication rate, including the need for further surgery, of surgical fixation with K-wires versus casting at 1-year post-randomisation.

3. To investigate, using appropriate statistical and economic analysis methods, the healthcare resource use and comparative cost-effectiveness at 1 year, of surgical fixation with K-wires versus plaster casting.

\section{Outcome measures}

The primary outcome measure for this study is the PRWE $^{7}$ at 12 months post-randomisation. The PRWE score $^{7}$ is a questionnaire designed specifically for assessment of distal radial fractures and wrist injuries that rates wrist function using a range of questions in two (equally weighted) sections concerning the patient's experience of pain and function. Scoring for all the questions is via an 11-point, ordered, categorical scale ranging from 'no pain' or 'no difficulty' (0) to 'worst ever pain' or 'unable to do' (10). Five questions relate to a patient's experience of pain and 10 relate to function and disability; scores for the 10 function items are summed and divided by 2 and added to the five pain items to give a score out of 100 (best score $=0$ and worst score $=100$ ). The PRWE score will be collected at baseline, 3, 6 and 12 months post-randomisation. The PRWE is the most sensitive outcome measure available for patients sustaining this specific injury. ${ }^{8}$

The secondary outcome measures in this trial are:

PRWE at 3 and 6 months, and area under the PRWE curve using data from all time points over the 12 months.

EQ-5D at 3, 6 and 12 months, and area under the EQ-5D curve over the 12 months using all time points: The EQ-5D-5L is a validated, generalised, HRQoL questionnaire consisting of five domains related to daily activities with a 5-level answer possibility, ${ }^{9}$ which will be converted into multiattribute utility score. ${ }^{10}$ This will be done according to the recommendation of the UK National Institute for Health and Care Excellence (NICE) at the time of finalising the statistical analysis plan (SAP).

Complications over 12 months: Complications recorded will include infection, and damage to nerves, tendons or blood vessels. Standard posterior-anterior and lateral radiographs will be taken at baseline 
(pre-reduction and post-reduction using the routine intraoperative fluoroscopic images from the operating theatre) and at the routine follow-up appointment 6 weeks after the injury. The radiographs will be assessed using the criteria described in Costa et al. ${ }^{11}$ Further surgical interventions, for loss of reduction/malunion or other complications after the index procedure, will be reported.

Healthcare resource use: Healthcare resource use will be monitored for the economic analysis. Unit cost data will be obtained from the latest available national databases such as the British National Formulary (BNF) and Personal Social Services Research Unit (PSSRU) Costs of Health and Social Care. ${ }^{12}$ The unit cost will be estimated in consultation with the Oxford University Hospitals finance department if the unit cost cannot be obtained from the national databases. The cost consequences following discharge including National Health Service (NHS) costs and patients' out-of-pocket expenses will be recorded via a short questionnaire which will be administered at 3, 6 and 12 months post-randomisation. Patient self-reported information on service use has been shown to be accurate in terms of the intensity of use of different services. $^{13}$

\section{Sample size}

The DRAFFT trial, ${ }^{4}$ which included the same patient population, estimated that the SD of the PRWE at 12 months was 16 . However, other studies of patients with a fracture of the distal radius showed SDs for the PRWE which was in the range $16-23$ points. ${ }^{14}$ Therefore, we have chosen a conservative estimate of the SD of 18 points.

The DRAFFT results also showed an approximate normal distribution for the PRWE scores at 12 months. ${ }^{4}$ A 6-point mean difference between groups equates to a standardised effect size of 0.33 , for an assumed SD of 18 points. MacDermid et al found that the PRWE is sensitive enough to detect subtle but clinically relevant changes in wrist function of this order of magnitude in patients sustaining a fracture of the distal radius. A mean difference in the PRWE of 6 points is just above the amount achieved if all the participants in one group responded they had one degree better response to any of the PRWE's constituent question (eg, one degree less difficulty in turning a doorknob) than the other group (each degree in response contributes 5 points to the overall score). We believe the target difference (6 points) would be important to patients on both an individual and a population level, and could lead to a change in clinical practice in the UK.

The total number of patients required to obtain a statistical power of $90 \%$ at the two-sided $5 \%$ significance level to detect a 6-point difference between groups for the primary outcome measure is 382 , that is, 191 patients will be required in each treatment group. Making a conservative allowance of just under $20 \%$ for lost to follow-up, we plan to recruit a minimum of 476 patients.

\section{Methodology}

Eligibility

Patients will be eligible for inclusion into the trial if:

- They have sustained a dorsally displaced fracture of the distal radius, which is defined as a fracture within $3 \mathrm{~cm}$ of the radiocarpal joint.

- They are over the age of 16 and able to give informed consent.

- The treating consultant surgeon believes that they would benefit from manipulation of the fracture.

Patients will be excluded from this trial if:

- The injury is more than 2 weeks old.

- The fracture extends more than $3 \mathrm{~cm}$ from radiocarpal joint.

- The fracture is open with a Gustilo grading greater than $1 .^{15}$

- The articular surface of the fracture (specifically the radiocarpal joint) cannot be reduced by indirect techniques. In a small number of fractures, the joint surface is so badly disrupted that the surgeon will have to open up the fracture in order to restore the anatomy.

- There is evidence that the patient would be unable to adhere to trial procedures or complete questionnaires, such as cognitive impairment.

\section{Consenting}

Recruitment will take place in 35-40 NHS trusts who treat patients with distal radius fractures in the UK. Eligible patients will be identified by the clinical team. The research associate will present the eligible patient with the participant information sheet and a verbal explanation of the trial procedures. The patients will then be given the opportunity to discuss any issues related to the trial with the research associate, as well as members of their family and friends.

In general, patients who are admitted with a fracture of the distal radius will have their treatment on the following day(s), so there will be sufficient time for the patients to consider taking part in the trial.

\section{Randomisation}

Those patients who consent to take part in the trial will have their treatment allocated using a secure, centralised, online randomisation service. The randomisation will occur after the manipulation of the fracture when the surgeon has determined that the fracture can be adequately reduced without the need to open the fracture to achieve reduction.

Although the great majority of these injuries are managed on planned trauma operating lists during normal working hours, the randomisation service will be open 24 hours each day to facilitate the inclusion of all potentially eligible patients. Randomisation will be on a 1:1 basis, stratified by centre, intra-articular extension of the fracture and age of the patient (above or below 50 years). The sequence will be generated by the trial statistician based at the Centre for Statistics in Medicine. 
Stratification by centre will help to ensure that any clustering effect related to the centre itself will be equally distributed in the trial arms. While it is possible that the surgeons at one centre may be more experienced in one or other treatment than those at another centre, all of the recruiting hospitals and indeed all hospitals throughout the NHS, use both techniques as part of their normal practice and generally, staff and surgeons will already be familiar with both forms of treatment. While this does not eliminate the surgeon-specific (such as a learning) effect of an individual at any one centre, ${ }^{16}$ the manipulation of a fracture of the distal radius is a common procedure so many surgeons will be involved in the management of this group of patients; between 10 and 30 surgeons at each centre, including both consultants and trainees. Therefore, it is anticipated that each individual surgeon will only operate on 2-3 patients enrolled in the trial, thus greatly reducing the risk of this surgeon-specific effect on the outcome.

Stratification on the basis of intra-articular extension of the fracture will address a major potential confounder, since disruption of this articular surface may predispose to secondary osteoarthritis of the wrist. ${ }^{17}$ Recent evidence ${ }^{18}$ suggests that other associated features of fractures of the distal radius which commonly appear in the classification systems, such as involvement of the ulnar styloid process, do not actually affect the functional outcome of the injury. Therefore, we would not propose to include any other fracture variables in the stratification of the randomisation.

Stratification on the basis of age will be used in an attempt to discriminate between younger patients with normal bone quality sustaining high-energy fractures, and older patients with low-energy (fragility) fractures related to osteoporosis. Empirically, both of these groups of patients could benefit, or not, from surgical fixation. However, the stratification may also help to identify any effect related to the quality of the patients' bone. The use of dual-energy X-ray absorptiometry is widely regarded as the gold standard for the assessment of bone density. However, such an investigation is not routinely available at all centres. Therefore, we propose to use age as a surrogate for bone density. In a large study in Norway involving 7600 participants, it was demonstrated that forearm bone mineral density remains stable up until the age of 50 years. After the age of 50 , bone mineral density decreased steadily in males, while in females there was an initial decline between the ages of 50 and 65 , with a further decline in the age groups thereafter. ${ }^{19} \mathrm{~A}$ study by Court-Brown et $a l^{20}$ assessed over a 1000 patients with a fracture of the distal radius. This study confirmed that there is a clear bimodal distribution for this type of fracture according to the age of the patient. The crossover of the two peaks of incidence was around 50 years of age. These studies provide strong evidence that patients over the age of 50 become increasingly vulnerable to fragility fractures of the distal radius. Therefore, we have chosen an age under and over 50 years as the stratification criteria for this trial. Furthermore, the study by Court-Brown et $a t^{20}$ demonstrated that in the UK, approximately $60 \%$ of patients sustaining a fracture of the distal radius were over 50 years, while $40 \%$ were younger than 50 years. The number of patients above and below this stratification age will, therefore, be similar (see the Analysis section).

\section{Pre and postrandomisation withdrawals}

Participants may decline to continue to take part in the trial at any time without prejudice. A decision to decline consent or withdraw will not affect the standard of care the patient receives. Once withdrawn, the patient will be advised to discuss their further care plan with their surgeon. On withdrawal of the patient, any data collected up until the time of withdrawal will be retained by the research team and included in the final analysis.

\section{Blinding}

The patients cannot be blind to their treatment as the wires have to be removed; usually in the outpatient clinic at the 6 weeks follow-up appointment. The treating surgeons will, of course, not be blind to the surgical/ non-surgical treatment they are providing. However, the treating clinical team will take no part in the postoperative assessment of the patients. The outcome data will be collected directly from the patient themselves.

\section{Trial treatments}

All of the participants will undergo a closed (without making any incisions in the skin) manipulation of the fracture. The manipulation will be carried out using either local, regional or general anaesthetic. The choice of anaesthetic will be left to the discretion of the treating surgeon/anaesthetist as per their normal practice.

As per routine clinical practice, an image intensifier $\mathrm{X}$-ray machine will allow the surgeon to judge that an adequate closed reduction has been achieved during the manipulation. The decision to accept the reduction will be left to the discretion of the treating surgeon, as per their normal practice. Only after this decision is made will the patient be randomised to one treatment or the other.

This trial will compare two techniques for holding the position of the bone fragments following a manipulation of a dorsally displaced fracture of the distal radius.

\section{Plaster cast}

This technique involves the application of a plaster cast which is shaped (moulded) over the skin to hold the bone fragments in position. This technique is simple and quick to perform, there is little risk of complications and the materials used are cheap. However, the plaster cast is not applied directly to the bone fragments and therefore it is possible for the bone fragments to redisplace under the cast, particularly when the swelling starts to settle a few days after the surgery. The principles of applying a moulded plaster cast are inherent in the technique, but in this pragmatic trial the type of casting material, extent of the cast and the details of the moulding technique will be left to the discretion of the 
treating surgeon as per their usual technique. Relevant information about the initial technique used and any subsequent interventions, such as cast replacement or removal, will be recorded.

\section{Surgical fixation with wires}

After the skin has been covered in antiseptic, sharp wires are passed through the skin over the back of the wrist and directly into the bone in order to hold the bone fragments in the correct position. The principles of K-wire fixation are also inherent in the technique, although there are several different options for the positioning of wires. The size and number of wires, the insertion technique and the configuration of wires will be left entirely to the discretion of the surgeon as per their normal practice. A plaster cast will be applied at the end of the procedure, as per standard surgical practice, but this does not need to be specifically moulded as the wires themselves hold the bone in position. Relevant information of the initial technique used and any subsequent interventions, such as metalwork removal, cast replacement or removal will be recorded.

\section{Rehabilitation}

All patients randomised into the two groups will receive the same standardised, written physiotherapy advice detailing the exercises they need to perform for rehabilitation following their injury. The written information was developed and tested by experienced physio/hand therapists as part of the original DRAFFT trial. All of the patients in both groups will be advised to move their shoulder, elbow and finger joints fully within the limits of their comfort. Patients will be asked to indicate if they had any other investigations/interventions as part of the 3, 6 and 12-month follow-up.

\section{Adverse event management}

Adverse events will be listed on the appropriate case report form for routine return to the 'DRAFFT2' central office. Serious adverse events (SAEs) will be entered onto the SAE reporting form and reported to the central study team. However, some adverse events which are foreseeable as part of the proposed treatment will not be reported on an SAE reporting form; they will be recorded on a complication form instead. These events include: complications of anaesthesia or surgery, for example, wound infection, damage to nerves, tendons or blood vessels, complex pain syndromes and thromboembolic events, and also further planned surgery for removal of symptomatic metalwork or for loss of fracture position/ malunion.

All participants experiencing SAEs will be followed up as per protocol until the end of the trial. All unexpected SAEs or Suspected Unexpected Serious Adverse Reaction (SUSARs) that occur between date of consent and 12 months follow-up point will be reported to the sponsor and ethics committee.

\section{End of trial}

The end of the trial will be defined as the collection/ receipt of the last follow-up questionnaire from the last participant.

\section{ANALYSIS}

\section{Statistical analysis}

Standard statistical summaries (eg, medians and ranges or means and variances, dependent on the distribution of the outcome) will be presented for the primary outcome measure and all secondary outcome measures. Baseline data will be summarised and visually compared with check comparability between treatment arms.

The primary outcome measure, PRWE score at 12 months after injury, will be compared between the two treatment groups on an intention-to-treat basis, which is according to the randomised groups irrespective of compliance with treatment allocation. A model which allows for clustering by centre will be used for the main analysis (ie, linear regression using cluster-robust SEs, implemented via the cluster option in Stata or equivalent). Although generally, we have no reason to expect that the clustering effects will be important for this study, the data will be hierarchical in nature, with patients naturally clustered into groups by recruiting centre and surgeon though this may not lead to tangible clustering of the primary outcome. ${ }^{21}$ Practice in DRAFFT, that has informed the design of this trial, was such that very few surgeons operated on more than one study participant. Surgeon clustering effects will not be included in the principal analysis of PWRE, which will be adjusted for centre and not surgeon. Other stratification variables (age and interarticular extension of the fracture) will be adjusted for in the main analysis as fixed effects as recommended to maintain the correct significance level. PRWE-based secondary outcomes (ie, PRWE at 3 and 6 months and area under the PRWE curve) will be analysed in the same manner.

It seems likely that some data may not be available due to voluntary withdrawal of patients, lack of completion of individual data items or general lost to follow-up. Where possible, the reasons for data 'missingness' will be ascertained and reported. Although missing data are not expected to be a problem for this study, the nature and pattern of the missingness will be carefully consideredincluding in particular whether data can be treated as missing at random. If judged appropriate, the impact of missing data will be explored with an approach such as a sensitivity analysis at least for the primary outcome. Reasons for ineligibility, non-compliance, withdrawal or other protocol violations will be stated and any patterns summarised.

Subgroup analyses of the two clinical stratifying variables (age and intra-articular extension) are planned. This will be undertaken for each of the stratifying variables using an extended model to formally test the interaction between each stratifying variable and the treatment 
factor; appropriate $95 \%$ CIs will be reported for the interaction effects in addition to $p$ values. These analyses will be labelled as exploratory and results from this analysis will be interpreted with due caution, and reported as such; in line with recommendations for subgroup analysis made elsewhere. ${ }^{22}$

The other (non-PRWE based) secondary outcomes will be analysed using a generalised linear model adjusting where relevant for stratification factors, and (for EQ-5D-5L outcomes), the corresponding baseline measurement.

All tests will be two sided and considered to provide evidence for a significant difference if the $p$ value is less than 0.05 (5\% significance level). Estimates of treatment effects will be presented with $95 \%$ CIs whenever possible. A detailed SAP will be agreed with the data and safety monitoring committee (DSMC) prior to the conduct of the study results analysis and unblinding of data to the clinical investigators. Any subsequent amendments to this initial SAP will be clearly stated and justified. Interim analyses will be performed only where directed by the DSMC. The statistical analysis is anticipated to be carried out using STATA (www.stata.com) and R (https://www. r-project.org/).

\section{Economic evaluation}

The economic evaluation will estimate the cost-effectiveness of plaster cast versus surgical K-wire in adults with a dorsally displaced fracture of the distal radius. Primary sources (eg, operating theatre records) will be used to record the duration of each procedure, theatre staffing, consumables, imaging, supplementary devices, postoperative recovery time and rehabilitation inputs. Community and social care service use will be collected at 3,6 and 12 months postrandomisation by using postal patient self-reported questionnaires. The data collected in the participant questionnaires at each time point will also record indirect costs and direct non-medical costs borne by participants and carers. Unit cost data will be obtained from the latest available national databases such as the BNF (https://www.medicinescomplete.com/mc/ bnf/current)and PSSRU Costs of Health and Social Care (http://www.pssru.ac.uk/project-pages/unit-costs).

HRQoL will be estimated using the EQ-5D-5L. ${ }^{9}$ Trial participants will be asked to complete EQ-5D-5L at baseline (preinjury and contemporary), 3, 6 and 12 months postrandomisation. Responses to the EQ-5D-5L will be converted into $3 \mathrm{~L}$ utility values using the algorithm approved by NICE at the time of the analysis. ${ }^{10}$

A within-trial evaluation will be conducted from a UK NHS and personal social services perspective using the DRAFFT2 trial data. The outputs of the cost-effectiveness analysis will be presented in terms of expected incremental cost-effectiveness ratios and cost-effectiveness acceptability curves generated via non-parametric bootstrapping as well as expected net monetary benefit.

Sensitivity analyses will include assessing the total cost of the trial from the societal perspective, impact of missing data and subgroup analyses. Costs from the societal perspective will consist of indirect costs (lost productivity of patients). The results of the multiple imputation method to address the impact of missingness in the base case analysis will be compared with the complete-case analysis. Similar to the statistical analysis, subgroup economic analyses of the two clinical stratifying variables (age and intra-articular extension) will be conducted.

\section{Trial oversight}

The day-to-day management of the trial will be the responsibility of the clinical trial manager, based at Nuffield Department of Orthopaedics, Rheumatology and Musculoskeletal Sciences and supported by the Oxford Clinical Trials Research Unit (OCTRU) staff. This will be overseen by the trial management group, who will meet monthly to assess progress.

A trial steering committee (TSC) and a DSMC will be set up. The DSMC will adopt a DAta MOnitoring. Committees: Lessons, Ethics, Statistics (DAMOCLES) charter which defines its terms of reference and operation in relation to oversight of the trial. They will not be asked to perform any formal interim analyses of effectiveness. They will, however, see copies of data accrued to date, or summaries of that data by treatment group and they will assess the screening algorithm against the eligibility criteria. They will also consider emerging evidence from other related trials or research and review related SAEs that have been reported. They may advise the chair of the TSC at any time if, in their view, the trial should be stopped for ethical reasons, including concerns about participant safety. DSMC meetings will be held at least annually during the recruitment phase of the study.

\section{Quality control}

The study may be monitored, or audited in accordance with the current approved protocol, relevant regulations and standard operating procedures by the host organisation, sponsor or appropriate regulatory authorities. A monitoring plan will be developed according to OCTRU standard operating procedures which involves a risk assessment. The monitoring activities are based on the outcome of the risk assessment and may involve central monitoring and site monitoring.

\section{Patient and public involvement}

A series of formal qualitative interviews were performed with patients suffering from fractures of the distal radius alongside the DRAFFT trial. ${ }^{11}$ The views and priorities of patients taking part in DRAFFT were used to inform the trial interventions and processes in this study.

To ensure ongoing patient and public involvement, a patient/carer representative will be actively involved in general trial management. In addition, a further independent patient/carer representative will become a member of the steering committee. 


\section{ETHICS AND DISSEMINATION}

The study monograph for the National Institute for Health Research Health Technology Assessment will be prepared by the trial management team on completion of the trial. A manuscript for a high impact peer-reviewed journal will be prepared simultaneously, which will allow for the results to be disseminated across the orthopaedic communities, the wider medical community, NICE and policy-makers. Authorship will be determined in accordance with the International Committee of Medical Journal Editors (ICMJE) guidelines and other contributors will be acknowledged. The results of this trial will substantially inform clinical practice on the clinical and cost-effectiveness of the treatment of this injury. The results of this project will be disseminated to patients via patient-specific newsletters and through local mechanisms at all participating centres.

\section{Author affiliations}

${ }^{1}$ Nuffield Department of Orthopaedics, Rheumatology and Musculoskeletal

Sciences, University of Oxford, Oxford, UK

${ }^{2}$ Department of Orthopedics, Leicester General Hospital, Leicester, UK

${ }^{3}$ Department of Orthopaedics, University Hospitals Coventry and Warwickshire NHS Trust, Coventry, UK

${ }^{4}$ Nuffield Department of Population Health, University of Oxford, Oxford, UK

Contributors JA: research methodology and management sections; first draft of manuscript. WS: sample size and statistical analysis. JD: background section; developed the research question. $\mathrm{HH}$ : background section; developed the research question. JAC: sample size and statistical analysis. MD: health economic evaluation. MEP: health economic evaluation. AG: health economic evaluation. SEL: background section; developed the research question. MLC: background section; developed the research question; research methodology and management sections. All authors reviewed and agreed the final manuscript.

Funding MLC/JAC/SEL/MD/AG/JD/HH obtained grant funding for this project. This project was funded by the UK NIHR HTA Programme (project number 15/27/01) and was supported by NIHR 0xford Biomedical Research Centre.

Competing interests MLC is a member of the UK National Institute for Health Research (NIHR) Health Technology Assessment (HTA) General Board; SEL is a member of the UK NIHR HTA Additional Capacity Funding Board, HTA end of life care and add-on studies, HTA Prioritisation Group and HTA Trauma Board. JAC was a member of the UK NIHR HTA Efficient Study Designs funding board.

Patient consent for publication Not required.

Ethics approval National Research Ethic Committee approved this study on the 6 October 2016.

Provenance and peer review Not commissioned; peer reviewed for ethical and funding approval prior to submission.

Open access This is an open access article distributed in accordance with the Creative Commons Attribution Non Commercial (CC BY-NC 4.0) license, which permits others to distribute, remix, adapt, build upon this work non-commercially, and license their derivative works on different terms, provided the original work is properly cited, appropriate credit is given, any changes made indicated, and the use is non-commercial. See: http://creativecommons.org/licenses/by-nc/4.0/.

\section{REFERENCES}

1. Barrett JA, Baron JA, Karagas MR, et al. Fracture risk in the U.S. Medicare population. J Clin Epidemiol 1999;52:243-9.

2. Chen NC, Jupiter JB. Management of distal radial fractures. J Bone Joint Surg Am 2007;89:2051-62.

3. Handoll HH, Madhok R. From evidence to best practice in the management of fractures of the distal radius in adults: working towards a research agenda. BMC Musculoskelet Disord 2003;4:27.

4. Costa ML, Achten J, Parsons NR, et al. Percutaneous fixation with kirschner wires versus volar locking plate fixation in adults with dorsally displaced fracture of distal radius: randomised controlled trial. BMJ 2014;349:g4807.

5. Costa ML, Jameson SS, Reed MR. Do large pragmatic randomised trials change clinical practice?: assessing the impact of the Distal Radius Acute Fracture Fixation Trial (DRAFFT). Bone Joint J 2016;98B:410-3.

6. NICE. Improving health and social care through evidence-based guidance. https://www.nice.org.uk/guidance/ng38

7. MacDermid JC, Turgeon T, Richards RS, et al. Patient rating of wrist pain and disability: a reliable and valid measurement tool. $J$ Orthop Trauma 1998;12:577-86.

8. MacDermid JC, Richards RS, Donner A, et al. Responsiveness of the short form-36, disability of the arm, shoulder, and hand questionnaire, patient-rated wrist evaluation, and physical impairment measurements in evaluating recovery after a distal radius fracture. J Hand Surg Am 2000;25:330-40.

9. Herdman M, Gudex C, Lloyd A, et al. Development and preliminary testing of the new five-level version of EQ-5D (EQ-5D-5L). Qual Life Res 2011;20:1727-36.

10. van Hout $B$, Janssen MF, Feng $Y S$, et al. Interim scoring for the EQ5D-5L: mapping the EQ-5D-5L to EQ-5D-3L value sets. Value Health 2012;15:708-15.

11. Costa ML, Achten J, Plant C, et al. UK DRAFFT: a randomised controlled trial of percutaneous fixation with Kirschner wires versus volar locking-plate fixation in the treatment of adult patients with a dorsally displaced fracture of the distal radius. Health Technol Assess 2015;19:1-124

12. Netten A. Unit cost of health and social care personal social services research unit. University of Kent: Canterbury, 1996.

13. Self-reports of health care utilisation: can a questionnaire replace a diary? 16th Annual meeting of the international society for health technology assessment in health care. The Netherlands: The Hague, 2000.

14. MacDermid JC, Roth JH, Richards RS. Pain and disability reported in the year following a distal radius fracture: a cohort study. BMC Musculoskelet Disord 2003;4:24 1471-2474.

15. Gustilo RB, Anderson JT. Prevention of infection in the treatment of one thousand and twenty-five open fractures of long bones: retrospective and prospective analyses. J Bone Joint Surg Am 1976;58:453-8.

16. Ramsay CR, Grant AM, Wallace SA, et al. Statistical assessment of the learning curves of health technologies. Health Technol Assess 2001;5:1-79.

17. Downing ND, Karantana A. A revolution in the management of fractures of the distal radius? J Bone Joint Surg Br 2008;90:1271-5.

18. Zenke $Y$, Sakai A, Oshige T, et al. The effect of an associated ulnar styloid fracture on the outcome after fixation of a fracture of the distal radius. J Bone Joint Surg Br 2009;91:102-7.

19. Berntsen GK, Fønnebø V, Tollan A, et al. Forearm bone mineral density by age in 7,620 men and women: the troms $\varnothing$ study, a population-based study. Am J Epidemiol 2001;153:465-73.

20. Court-Brown CM, Rimmer S, Prakash U, et al. The epidemiology of open long bone fractures. Injury 1998;29:529-34.

21. Cook JA, Bruckner T, MacLennan GS, et al. Clustering in surgical trials-database of intracluster correlations. Trials 2012;13:2.

22. Brookes ST, Whitley E, Peters TJ, et al. Subgroup analyses in randomised controlled trials: quantifying the risks of false-positives and false-negatives. Health Technol Assess 2001;5:1-56. 